Voix et Images

volxetimages

\title{
Prolégomènes à une méthodologie d'analyse des réseaux littéraires. Le cas de correspondance de Henri-Raymond Casgrain
}

\section{Manon Brunet}

Volume 27, numéro 2 (80), hiver 2002

La sociabilité littéraire

URI : https://id.erudit.org/iderudit/290053ar

DOI : https://doi.org/10.7202/290053ar

Aller au sommaire du numéro

\section{Éditeur(s)}

Université du Québec à Montréal

\section{ISSN}

0318-9201 (imprimé)

1705-933X (numérique)

Découvrir la revue

Citer cet article

Brunet, M. (2002). Prolégomènes à une méthodologie d'analyse des réseaux littéraires. Le cas de correspondance de Henri-Raymond Casgrain. Voix et Images, 27(2), 216-237. https://doi.org/10.7202/290053ar

\section{Résumé de l'article}

L'analyse des réseaux littéraires a ses particularités. Or, les études littéraires ne disposent pas encore d'une méthodologie d'analyse d'ensemble qui réponde aux questionnements sociohistoriques singuliers que posent les réseaux littéraires par rapport aux groupes ou aux institutions littéraires. La correspondance de Henri-Raymond Casgrain, chef du réseau littéraire québécois de la deuxième moitié du XIX ${ }^{\mathrm{e}}$ siècle, quantitativement et qualitativement très riche (5,000 lettres échangées avec 850 correspondants d'un réseau international d'intellectuels très actifs), nous aide à jeter les bases d'une méthodologie d'analyse. Ainsi, nous pouvons mieux comprendre comment délimiter le territoire géographique, idéologique, spatial et temporel d'un réseau, ses zones de pouvoir et d'action, les activités des leaders et des membres, et ultimement comment apercevoir « le littéraire » au sein d'un réseau nécessairement polyfonctionnel. 


\title{
Prolégomènes à une méthodologie d'analyse des réseaux littéraires. Le cas de la correspondance de Henri-Raymond Casgrain*
}

\author{
Manon Brunet, Université du Québec à Trois-Rivières
}

\begin{abstract}
L'analyse des réseaux littéraires a ses particularités. Or, les études littéraires ne disposent pas encore d'une méthodologie danalyse d'ensemble qui réponde aux questionnements sociobistoriques singuliers que posent les réseaux littéraires par ropport aux groupes ou aux institutions littéraires. La correspondance de Henri-Rạınond Casgrain, cbef du réseaul littéraire québécois de la denxième moitié du $X I X^{2}$ siècle, quantitativement et qualitativement très riche (5,000 lettres échangées alec 850 correspondants d'un réseau international d'intellectuels très actifs), nous aide à jeter les bases d'une méthodologie d'analyse. Ainsi, nous poutons mieux comprendre comment délimiter le territoire géographique. idéologique, spatial et temporel d'un réseau, ses zones de pouvoir et daction, les activités des leaders et des membres, et ultimement comment apercèoir "le littéraire" au sein diun réseau nécessairement polyfonctionnel.
\end{abstract}

Délimiter le territoire, interpréter et caractériser les activités d'un réseau spécifique sont des entreprises théoriques et sociohistoriques périlleuses. La nature et les fonctions d'un réseau composé d'individus, de groupes sociaux ou d'institutions sont presque aussi variées que le nombre et le type de réseaux eux-mêmes. Certes, les mathématiques, l'anthropologie et la sociologie peuvent fournir des concepts d'analyse et des outils de représentation graphique des réseaux. Les récents ouvrages de Vincent Lemieux résument bien la question'. Cependant, quand il s'agit

* Cette recherche est subventionnée par le Conseil de recherches en sciences humaines et l'Université du Québec à Trois-Rivières.

1. Vincent Lemieux, Les réseaux d'acteurs sociatux, Paris, Presses universitaires de France, 1999,146 p.; id., À quoi sernent les réseanx socianx?, Québec, Institut québécois de recherche sur la culture, $2000,109 \mathrm{p}$. 
d'observer le phénomène dans le domaine culturel, tel le réseau littéraire, des difficultés supplémentaires se présentent rapidement. Cela explique pourquoi si peu d'ouvrages osent s'attaquer de front à la problématique des réseaux littéraires, contrairement aux nombreux écrits consacrés à la question institutionnelle.

Nous voudrions montrer comment nous avons abordé quelques-unes de ces difficultés à partir du cas de la correspondance de l'animateur de la littérature nationale du Québec au $\mathrm{XIX}^{\mathrm{e}}$ siècle, Henri-Raymond Casgrain $^{2}$. Cette riche correspondance, en cours d'édition au Projet Casgrain, est extrêmement instructive sur la façon de saisir à vif, en circuit fermé, le fonctionnement d'un réseau littéraire, et en particulier celui de Casgrain, pendant un demi-siècle, soit de 1851 à 1904 . Mis à part deux articles succincts datant de 1943 et de 1954, année du cinquantenaire de la mort de Casgrain, cette correspondance n'a pas fait l'objet d'une présentation d'ensemble ${ }^{3}$.

\section{Observations préliminaires}

Les réseaux culturels sont particulièrement porteurs de changements sociaux. Alors que les réseaux de parenté reproduisent la tradition, que les réseaux politiques cherchent à ancrer leurs pouvoirs par la fondation et le maintien coercitif d'institutions et que les réseaux économiques défendent plus des intérêts individuels et corporatifs que collectifs. La créativité qui caractérise les réseaux culturels est due à leur plus grande ouverture sur l'externe, à leur perméabilité aux différences dans et entre les sociétés ainsi qu'à la recherche de la nouveauté par l'avancement des connaissances ou l'usage illimité de l'imaginaire humain. Par conséquent, le territoire d'un réseau littéraire est toujours plus étendu et ouvert. Ce qui rend son observation plus difficile. La mondialisation des échanges actuels, due au développement des nouvelles technologies d'information, ne fait qu'accentuer le phénomène dans tous les types de réseaux, à commencer par les réseaux artistiques où les langages linguistiques et visuels servent à la fois de moyens et de finalités de communication.

On ne saurait limiter le territoire d'un réseau littéraire ni par le type de profession qu'exercent ses membres ni par leur lieu d'origine et encore moins par le genre littéraire auxquel ils s'adonnent. Ces critères n'expliquent pas ce qui relie fondamentalement les écrivains entre eux. Les études sociologiques en littérature, qui ont tenté de dresser un portrait

2. Les neuf volumes de la correspondance paraîtront aux Presses de l'Université Laval. Le premier sortira à l'automne 2002.

3. Pascal Potvin et Arthur Maheux, "Les correspondants de l'abbé Henri-Raymond Casgrain ", Mémoires de la Société royale du Canada, Section 1, 1943, p. 79-88; Honorius Provost, "L'abbé Henri-Raymond Casgrain et ses relations d'amitié ", Revue de l'Université Laval, vol. VIII, n' 9, mai 1954, p. 791-810. 
des écrivains selon ces critères, plus ou moins sociaux ou littéraires, ont au mieux permis l'observation de cohortes, de générations littéraires inscrites à l'intérieur d'une simple chronologie ou d'une hiérarchie des classes sociales ou des genres et que l'histoire littéraire appelle des groupes, des mouvements littéraires ou des littératures nationales pour des commodités pédagogiques de classement ${ }^{4}$. Or, l'étude d'un réseau littéraire permet de saisir les motivations et les lieux d'activité que partagent réellement des écrivains.

Les écrivains et leurs œuvres sont soumis à des influences d'allégeances esthétiques et idéologiques extrêmement variées et variables. Toutes lectures et écritures y sont subordonnées. Leurs motivations et leurs lieux d'activité partagent donc un territoire qui dépasse souvent les contraintes liées à l'appartenance à une seule classe sociale, à un seul genre littéraire, à une seule représentation du littéraire, à une seule région, voire à un seul pays. L'activité créatrice supporte mal les contraintes, y compris les siennes propres. Au sein d'un réseau littéraire, la variété et la variation des pratiques sont valorisées. Autrement, l'œuvre est discréditée; on parle de plagiat. La censure, à ce titre, provenant des réseaux littéraires eux-mêmes est implacable. Elle s'observe depuis l'Antiquité, avant même que les droits d'auteur ne fassent l'objet de législations nationales et internationales. Depuis la fin de la rhétorique, la modernité littéraire exige encore plus d'originalité des œuvres littéraires, de telle sorte que leur classement devient presque impossible.

Cette distance prise avec les contraintes, grâce au jeu littéraire, est la condition première d'exercice de toute littérature. Autrement, la "littérature" serait une simple transposition de la réalité, volontaire ou imposée, résultat d'une activité d'écrivant, selon la terminologie de Barthes. Ainsi, les réseaux culturels enjambent les temps et les espaces sociaux de la manière la plus désordonnée, au risque de perdre de la légitimité sociale mais jamais leur pouvoir de créativité, de transformation sociale, même quand ils nous semblent conservateurs, embrigadés ou surveillés. Tout intellectuel a la prétention de changer le monde, qu'il soit présent, passé ou à venir. L'usage de la liberté de parole et d'action est l'enjeu des réseaux culturels. Les réseaux de droite, autoritaires, jouissent de cette liberté tout entière et sans opposition. En revanche, plus les réseaux de gauche refusent l'autorité, toute espèce de doxa, plus ils acceptent de partager cette liberté. En dernière instance, on conviendra que la droite ne se distingue pas de la gauche par le seul contenu des idées défendues, mais bien par l'usage pratique qui en est fait dans l'activité sociale. Ainsi, l'on pourra qualifier de droite certains réseaux marxistes qui imposèrent

4. Christophe Charle, "L'expansion et la crise de la production littéraire, $2^{\mathrm{e}}$ moitié du $19^{\mathrm{e}}$ siècle", Actes de la recherche en sciences sociales, $\mathrm{n}^{\prime \prime} 4$, juillet 1975, p. 44-65; id., "Situation du champ littéraire", Littérature, n 44, décembre 1981, p. 8-20. 
leurs vues au point de réduire au silence des générations d'écrivains; et de gauche, des pontificats qui, par des efforts de décentralisation et d'ouverture, accommodèrent concrètement les pratiques religieuses aux changements sociaux ambiants, à commencer par le rituel religieux lui-même, et réduisirent la liberté d'action de leurs instances de censure sur les œuvres intellectuelles.

Dans un tel chaos d'idées et de pratiques, comment délimiter le territoire d'un réseau désireux de s'affranchir constamment de son territoire d'origine afin de, paradoxalement, mieux l'afficher aux yeux du monde? Comment observer le fonctionnement d'un réseau à une si grande échelle? Comment y distinguer les pratiques proprement littéraires parmi les multiples activités auxquelles s'adonnent les écrivains?

\section{Le territoire dans son ensemble}

Le réseau de Henri-Raymond Casgrain se révèle à travers sa correspondance de 5000 lettres ${ }^{5}$ envoyées et reçues avec ses 850 correspondants provenant des cinq continents :

$\begin{array}{lc}\text { Répartition géographique du réseau de correspondants } \\ \text { de Henri-Raymond Casgrain, } 1851-1904 \\ \text { Canada (Prov. de Québec) } & 59 \% \\ \text { France } & 22 \% \\ \text { États-Unis } & 8 \% \\ \text { Canada anglais } & 5 \% \\ \text { Canada (Acadie) } & 3 \% \\ \text { Europe (hors France) } & 3 \% \\ \text { Afrique } & 1 \% \\ \text { Asie } & , 05 \% \\ \text { Australie } & , 05 \%\end{array}$

L'étendue de ce réseau dépasse de très loin le territoire de l'École littéraire de Québec dont Casgrain aurait été le chef de file et l'animateur, selon l'histoire littéraire. Même si l'on imagine que les activités du réseau à Québec n'apparaissent peut-être pas autant qu'elles le devraient dans cette répartition fondée sur les échanges épistolaires, en tenant compte du fait que plus les membres d'un réseau sont à proximité les uns des autres, moins ils ont besoin d'échanger de lettres (ce qui était le cas de Casgrain, Crémazie, J.-B.-A. Ferland, P.-J.-O. Chauveau, Charles-Honoré Laverdière, Hubert LaRue, J.-C. Taché, Antoine Gérin-Lajoie et Alfred Garneau avant le déménagement du Parlement à Ottawa), il est néanmoins clair que l'on ne peut se confiner au seul territoire de cette ville pour saisir l'étendue réelle des activités d'un tel réseau national et international.

5. Cela comprend les 4000 lettres environ envoyées à Casgrain et conservées dans le Fonds Casgrain des Archives du Séminaire de Québec au Musée de la civilisation de Québec, de même que le millier de lettres de Casgrain que nous avons localisées dans de nombreux fonds d'archives privés et publics canadiens et étrangers. 


\section{La zone d'influence principale}

Néanmoins, rien n'empêche de voir une ou des concentrations d'activités sur des parties de territoire du réseau qui n'apparaissent pas au premier coup d'œil quand on examine ainsi de haut une correspondance. Le matériau utilisé n'est pas en cause, mais bien la perspective qu'on adopte. Si l'on s'en tient à une seule répartition géographique des correspondances, on peut facilement perdre de vue des concentrations qui indiqueront les véritables lieux géographiques ou institutionnels d'activités, formels ou informels d'influence, quand bien même leur représentativité dans le tableau général reste fiable. Ainsi, le réseau des tiers mentionné dans chacune des lettres est le pius révélateur des zones géographiques ou intellectuelles d'influence d'un réseau, et situe du coup les membres les plus influents.

Dans le cas du réseau de Casgrain, ces tiers appartiennent en majorité à des groupes institutionnels (Collège de Sainte-Anne-de-la-Pocatière, Université Laval, Séminaire de Québec, Archevêché de Québec, Parlement à Québec et plus tard à Ottawa), semi-formels (collaborateurs aux revues littéraires comme Le Foyer canadien, journalistes à L'Opinion publique), ou informels (l'arrière-boutique de Crémazie, le presbytère de Beauport ou de Notre-Dame-de-Québec où réside Casgrain, le Manoir d'Airvault à Rivière-Ouelle où Laure Conan, comme bien d'autres, ira rencontrer Casgrain, le Cercle des Dix à Ottawa) de Québec, de la Côte-du-Sud et d'Ottawa. Les activités littéraires dont il est question dans les lettres ont lieu principalement dans ces zones, nonobstant le nombre de lettres qui nous parviennent de ces zones, puisque si l'on se fie uniquement au tableau général ci-haut, la zone québécoise, par exemple, ne représenterait qu'un peu plus que l'ensemble de toutes les autres zones géographiques, alors que les occurrences à des membres ou à des activités québécoises dans les lettres sont bien supérieures à $59 \%$.

\section{Les zones périphériques ou les relais}

Après vérification des mentions de tiers dans les lettres, on pourra aussi conclure qu'avec une représentativité quantitative de $22 \%$, la zone française fait bel et bien partie du réseau, mais qu'elle est en périphérie de la zone centrale d'influence québécoise. Nous voilà donc face à cette réalité propre aux réseaux culturels dont nous parlions plus haut: le territoire d'un réseau littéraire est très étendu parce qu'il nécessite obligatoirement une ouverture sur d'autres littératures. Cette ouverture est réelle, les relations sont très nombreuses et actives entre la zone québécoise du réseau de Casgrain et Rameau de Saint-Père, et des membres de l'Académie française, tel Xavier Marmier, et des écrivain(e)s de gauche ou de droite (Gaume ou la féministe Clarisse Coignet), et de nombreux archivistes, canadianistes et descendants des familles de la Nouvelle-France 
(les Lévis, Montcalm), et des éditeurs et libraires français comme Tours et Bossange. Cependant, ces relations ne donnent pas souvent lieu à des activités qui répondent aux besoins collectifs du réseau. C'est pourquoi ces activités sont moins citées dans la correspondance.

L'ouverture ainsi créée a quand même ses limites protectionnistes. Les zones plus éloignées servent de relais, plutôt que de point d'ancrage, de départ et d'arrivée, relais entre ce que désirent réaliser au mieux les membres de la zone principale du réseau et ce qui en résultera effectivement. Ici, par exemple, les membres de la zone française du réseau de Casgrain permettent aux écrivains québécois d'avoir accès à de l'information privilégiée dans les archives privées ou publiques concernant leur histoire; ce qui leur donne les moyens d'écrire, sous toutes ses formes (poétique, historique, romanesque, essayiste, dramatique, etc.), la fameuse littérature nationale dont ils se réclament. De même, les éditeurs, les libraires et l'Académie française serviront de ponts entre les auteurs publiés et les écrivains légitimés au Canada même. Vincent Lemieux affirme que des ponts très forts et incontournables sont souvent créés entre des individus ou des blocs d'individus qui font en réalité peu de choses ensemble. C'est ce qu'il appelle al'utilité des liens faibles ${ }^{6}{ }$. Des liens faibles offrent la possibilité d'être "branché "via l'externe à de l'information qui ne circulerait pas dans un réseau trop fermé où tous les écrivains se connaissent et s'échangent des sources similaires voire identiques d'information, de lectures, d'édition, de promotion, etc. D'après Lemieux, ce sont bien souvent des leaders dans leur zone d'influence principale respective qui prennent l'initiative et la charge de ces relais, renforçant l'influence générale de leurs réseaux respectifs dans leur propre milieu social et auprès des autres. Le réseau littéraire français a joué ce rôle dans le développement du réseau littéraire québécois, jusqu'à aujourd'hui.

\section{Les zones de pouvoir ou les leaders}

Après avoir observé des zones d'influence, il est nécessaire de déterminer le degré d'influence d'un membre au sein du réseau. Prenons le cas d'Octave Crémazie. Même si nous n'avons aucune lettre échangée entre Casgrain et le poète-libraire de Québec avant l'exil de ce dernier en 1862, les mentions de Crémazie comme tiers par les divers correspondants, entre 1851 et 1862, sont suffisantes pour que nous voyions l'influence réelle des activités de production et de diffusion littéraires de Crémazie sur l'ensemble du réseau et non seulement sur l'individu Casgrain. En revanche, les 14 lettres (12 envoyées et 2 reçues) qui suivent l'exil montrent l'isolement, la perte réelle de l'influence de Crémazie au sein du réseau de Casgrain, car il s'agit d'un échange

6. Vincent Lemieux, À quoi servent les réseaux sociaux?, op. cit., p. 36. 
strictement bipolaire et Crémazie n'est presque plus cité dans les lettres des autres correspondants. Ces lettres ne provoquent aucun échange littéraire entre tiers, sauf avec le libraire Bossange chez qui se réfugie Jules Fontaine. Isolées, elles viennent essentiellement confirmer la nouvelle position que Casgrain occupe au sein du réseau après le départ de Crémazie, soit celle de chef de file. D'ailleurs, en exil, Crémazie cesse quasiment toute activité d'écriture. Il devient alors à titre pré-posthume un modèle littéraire inscrit dans la mémoire collective, le "poète national ", une référence mais sans plus, potentiellement "activable" comme le deviendra bientôt F.-X. Garneau lui-même, l'" historien national ". Voilà un exemple percutant de la nécessité de ne pas se fier à la seule répartition nominative ou géographique des correspondants afin de mesurer et l'étendue d'un territoire et l'étendue de l'influence d'un membre du réseau au sein d'un territoire.

Notons encore qu'un réseau gagne à n'être pas sous l'influence d'un seul leader. Chaque zone du territoire a, bien sûr, son leader. Il (elle) a, en fonction de son territoire, les mêmes pouvoirs de parole et d'action que le leader du réseau dans son ensemble, soit de promouvoir une cause commune, d'encourager chacun à la promouvoir selon ses talents, de s'assurer des résultats obtenus par la défense de cette cause et tout ceci en s'exposant d'abord comme modèle lui-même. Cependant, le chef du réseau a des responsabilités supérieures: il doit définir cette cause, fournir les moyens de la réaliser, coordonner l'ensemble des zones d'activité et récompenser ceux et celles qui l'auront fait progresser. Dans un réseau littéraire, ces responsabilités consistent à définir un programme littéraire, à créer un réseau de contacts le plus fiable et étendu possible afin d'assurer l'accès à tous les moyens (de production, de diffusion et de légitimation littéraires) nécessaires à la réalisation du programme, à coordonner, notamment par la correspondance, ce réseau plus ou moins proche de la zone centrale et à jouer le rôle de critique littéraire de référence.

C'est exactement ce à quoi s'emploie Casgrain quand, en 1866, il publie dans Le Foyer canadien son article programmatique "Le mouvement littéraire en Canada ${ }^{7}$, au même moment, comme par hasard, où la revue révise son programme éditorial sous le titre de "Nouveau Prospectus $^{8}$ ". Les abonnés reprochaient au Foyer canadien de ne servir, depuis 1863, qu'à la réédition de textes nationaux "éparpillés dans les gazettes de 1850 à 1860 ", "compilation faite avec soin, pour servir de continuation au

7. Henri-Raymond Casgrain, "Le mouvement littéraire en Canada ", Le Foyer canadien, vol. IV, 1866, p. $1-31$.

8. La Direction du Foyer canadien, "Nouveau Prospectus", ibid., p. i-viii. Nous renvoyons à cette référence pour toutes les citations suivantes, extraites de ce nouveau prospectus. 
Répertoire national" de J. Huston, mais qui ne montrait pas, sauf exception, les nouvelles œuvres littéraires créées par le réseau activé depuis 1860. De plus, les souscripteurs "se plaignent que [le] Recueil manque de variété". Sous l'influence de Casgrain, seront effectués des changements majeurs de contenu, de format, de choix d'éditeur et de prix d'abonnement. D'abord sur le plan du contenu, la revue comprendra désormais trois parties: une réservée aux productions des "nouveaux venus" dans "le mouvement littéraire qui s'est produit dans la jeunesse instruite"; une seconde, "se composera d'un choix de littérature française contemporaine "; une dernière, sera une "revue mensuelle où seront relatés les événements politiques et littéraires de quelque importance de l'ancien et du nouveau monde, d'une revue critique des ouvrages nouveaux": "rien ne sera négligé pour donner l'attrait à ces dernières pages et en faire la chronique amusante de la littérature contemporaine". Ensuite, le format change, triplant le nombre de pages de chaque livraison (de 32 à 96 pages); l'imprimeur gouvernemental Desbarats ayant dû suivre le récent déménagement du Parlement à Ottawa, $\mathrm{C}$. Darveau prend la relève et est chargé de nouvelles fonctions, comme la perception des abonnements, la correspondance avec les agents locaux de distribution, sous le nouveau titre de "Gérant du Foyer". Finalement, le prix de l'abonnement double, passant de $1 \$$ à $2 \$$. Or, ce qui caractérise toutes ces modifications, c'est la priorité accordée désormais à étendre le réseau tant sur le plan de l'organisation structurelle que sur le plan idéologique. Cette expansion se fait au nom de la contemporanéité. La littérature nationale ne sera plus désormais une simple mémorisation et commémoration des œuvres du passé, elle ouvrira ses portes aux nouvelles productions locales, mais aussi à la littérature française contemporaine, aux événements politiques nordaméricains et européens modernes et, enfin, elle commencera à se doter d'une instance de légitimation indispensable au contrôle de cette ouverture sur tant de nouveautés, la critique littéraire. À ce titre, 1866 marque un tournant important dans l'histoire du réseau littéraire québécois qui bénéficiera jusqu'au début du $\mathrm{xx}^{\mathrm{e}}$ siècle d'un dynamisme moderne qui n'a rien à voir avec un repli sur soi.

Le leadership de Casgrain s'affirme dans une centaine d'autres articles de lui, qui mériteraient d'être davantage connus aujourd'hui ${ }^{9}$, car on u constaterait que le programme littéraire du réseau est régulièrement mis à jour. De même quand Casgrain façonne un réseau multiculturel et multifonctionnel (où toutes les fonctions de la division du travail littéraire sont représentées: du copiste au critique littéraire), l'entretient et l'élargit par son abondante correspondance, par des rencontres ou ses nombreux voyages (15 en Europe, 1 au Moyen-Orient, 7 en Ontario et aux États-

9. Nous avons retracé une centaine d'articles de critique littéraire de Casgrain que l'on ne peut pas énumérer ici. La liste en sera fournie dans l'édition' de la correspondance. 
Unis, 1 à Cuba, 4 en Acadie et dans les Provinces maritimes ${ }^{10}$ ); quand il s'assure que toutes les zones du réseau sont en contact les unes avec les autres et qu'il y ait le moins possible d'écrivains isolés (ex. Octave Crémazie, Apollinaire Gingras, Laure Conan) et quand, finalement, il récompense par sa critique les écrivains de la littérature nationale, qu'il leur ouvre la porte de l'Académie française et qu'il leur offre en 1882, comme membre fondateur, la Société royale du Canada, instance de légitimation suprême au pays même. Sans toutes ces précautions, le réseau de Casgrain n'aurait sûrement pas tenu le coup pendant un demi-siècle.

Il semble bien qu'aucun autre écrivain québécois n'ait eu autant d'influence de son vivant, pendant une aussi longue période, dans toute l'histoire littéraire québécoise. Casgrain n'a pas publié autant que l'historien Benjamin Sulte, n'a pas le talent poétique de Crémazie ou de Fréchette, et n'obtient pas au pays le succès populaire du romancier Joseph Marmette, mais il a écrit 85 ouvrages, plus de 200 articles, a fait publier, en l'espace de 10 ans, 80000 volumes des membres de son réseau en tant qu'éditeur intellectuel de la série nationale de livres de récompense ${ }^{11}$, et il fut l'écrivain du XIX ${ }^{e}$ siècle le plus édité et le plus lu à l'étranger: 9 éditions chez Mame \& Fils à Tours des Français au Canada, 5 éditions des Héros de Québec, 4 éditions de Montcalm et Lévis, une traduction allemande de son Histoire de Mère Marie de l'Incarnation ${ }^{12}$, sans compter des articles aux États-Unis. Seulement pour Montcalm et Lévis, Casgrain récolta 60 articles de critique venant de sources aussi variées que The Atlantic Montbly de New York/Boston, le Bulletin du Syndicat central de Paris, La Touraine républicaine de Tours, le Journal du Loiret d'Orléans, Le Soleil du Midi de Marseille, Le Patriote de Normandie de Rouen, Le Correspondant de Paris,

10. Entre 1858 et 1899 , Casgrain effectue 28 grands voyages dune durée moyenne de quatre mois, en Europe $(1858,1867,1873,1885-1886,1887,1887-1888,1888-1889$, $1890,1890-1891,1892-1893,1893-1894,1895,1896-1897,1897-1898,1898-1899)$, au Moyen-Orient (1891-1892), aux États-Unis ou en Ontario $(1868,1869,1871,1880,1881$, $1882,1885)$, à Cuba (1885) et en Acadie ou dans les Provinces maritimes $(1885,1887$, 1894, 1896).

11. En allant rigoureusement aux sources, Jean-Paul Hudon, auteur de la thèse de doctorat sur L'abbé Henri-Raymond Casgrain, l'bomme et l'xuvre (Ottawa, Université d'Ottawa, 1978,638 p.), a tout récemment corrigé la fausse idée que lon se faisait de Casgrain comme "profiteur" dans ce travail éditorial. Voir son article "Henri-Raymond Casgrain, Gédéon Ouimet et les livres donnés en prix dans les écoles de 1876 à 1886", Voix et images, vol. XXVI, n"3 (78), printemps 2001, p. 596-616. La correspondance privée de Casgrain que nous éditons confirme ces conclusions.

12. Henri-Raymond Casgrain, Les Français au Canada, Tours, Mame \& Fils, 1895, 1898, 1909, 1913, 1924, 1926, 1931, 1932, 1936; Les Héros de Québec, Tours, Mame \& Fils, 1895, 1899, 1913, 1927, 1931 ; Guerre du Canada: Montcalm et Lévis, Tours, Mame \& Fils, 1898, 1899, 1926, 1931; Gescbichte der Mutter Maria von der Menschwerdung, ersten Oberin der Ursulinerinnen in Canada, 1599-1672, mit einer gescbichtlichen Einleitung über die Gründung dieser Colonie, autorisierte Uebersetzung nach der zweiten französ, Regensburg/New York/Cincinnati, Papier, Drud und Verlag von Friedrich Pustet, 1872. 
Le Journal du Maine et Loire d'Angers, L'Écho du Nord de Lille, Le Journal des débats de Paris, Le Moniteur de l'Ariège de Foix, L'Univers et le monde de Paris, Le Journal des Demoiselles de Paris, La Nation de Paris, Le Figaro de Paris, La Dépêche de Besançon, Bookseller de Londres, L'Indépendance belge de Bruxelles, etc. ${ }^{13}$ Toutes proportions gardées et en terme d'étenchue spatiale et temporelle de réseau, de diversité d'activités littéraires réalisées, il faut admettre que l'influence de Casgrain au Québec est comparable, au même moment, à celle de Hugo en France, en tant que modèle romantique et que leader littéraire. Par conséquent, il faudrait parler de Casgrain plutôt comme chef du réseau québécois, entre 1850 et 1900 , et pas uniquement de la zone centrale appelée "École littéraire de Québec" ni seulement pour les années 1860 qui correspondent, en fait, aux tout débuts de l'activation de ce réseau.

Or, toutes ces tâches sont très lourdes pour un seul individu. Par conséquent, Casgrain ne sera pas le seul leader de ce réseau littéraire très étendu, même s'il en restera le dominant jusqu'à sa mort. Son principal conseiller littéraire sera le fils de l'historien national, Alfred Garneau, décédé par hasard en 1904, un mois après Casgrain... Isabelle Lefebvre, dans Henri-Raymond Casgrain épistolier: réseau et littérature au $19^{\mathscr{}}$ siècle, a très bien montré que cette alliance est une "histoire du littéraire qui s'écrit “à la vie, à la mort" 14 ". Il reste à analyser, en détail, comment opère Alfred Garneau auprès des autres écrivains que Casgrain; ce qui fait présentement l'objet du mémoire de Caroline Vallée ${ }^{15}$. Mais d'ores et déjà, si l'on se réfère à ses très nombreuses mentions comme tiers dans la correspondance du réseau, il est manifeste que Garneau a autant d'influence, sinon davantage, que Casgrain sur le contenu et la forme des œuvres littéraires. Par exemple, il révise non seulement l'Histoire $d u$ Canada de son défunt père et les épreuves d'un grand nombre de publications, mais il fournit des informations historiques à Joseph Marmette pour ses romans, inspire Casgrain clans le contenu de ses conférences, corrige les poésies des uns et des autres, etc. Cependant, Casgrain demeure celui qui contrôle les moyens de diffusion et de légitimation des œuvres littéraires, au centre comme en périphérie de son réseau.

13. Il serait trop long de donner ici toutes les références complètes à ces articles, mais sachons qu'ils parurent en 1897 et 1898 . Dáns l'édition de la correspondance, on trouvera tous les détails bubliographiques.

14. Isabelle Lefebvre, "Henri-Raymond Casgrain et Alfred Garneau: une histoire du littéraire qui s'écrit "à la vie, à la mort ", Manon Brunet, Vincent Dubost, Isabelle Lefebvre, Marie-Élaine Savard, Henri-Raymond Casgrain, épistolier: réseau et littérature au $19^{\prime}$ siècle, Québec, Nuit blanché éditeur, 1995, p. 81-151. Toute la publication est consacrée à des leaders de différentes zones socioculturelles du réseau de Casgrain, soit: le Canadien français, Alfred Garneau, le Français Rameau de Saint-Père, l'Américain Francis Parkman et le Canadien anglais George MacKinnon Wrong.

15. Caroline Vallée, assistante de recherche au Projet Casgrain, dont le mémoire de maîtrise est en cours au Département de français de l'UQTR. 
L'influence d'Alfred Garneau ne déborde pas la zone centrale du réseau. D'autres leaders ont la charge d'autres zones de regroupement stratégiques du réseau : Francis Parkman (pour la zone américaine), Rameau de Saint-Père (pour la zone française), George Mackinnon Wrong (pour la zone canadienne-anglaise), Placide Gaudet (pour la zone acadienne). Tous sont, comme Casgrain, des historiens de la Nouvelle-France et les leaders principaux dans leurs réseaux respectifs. Au sein du réseau de Casgrain, ils correspondent tous non seulement avec Casgrain mais aussi entre eux, dans tous les sens; ce qui démontre bien l'efficacité de la coordination assurée par le leader principal, Casgrain. Alfred Garneau ne correspond pas avec eux, appuyant doublement le fait que c'est bel et bien Casgrain qui, en créant des ponts entre les zones périphériques, confirme son statut de chef dans ce grand réseau.

\section{Les luttes de pouvoir}

Dans un réseau, les luttes de pouvoir sont différentes de celles au sein de l'institution littéraire, telles qu'elles sont décrites, par exemple, chez Jacques Dubois ${ }^{16}$. La raison en est simple: le réseau est un système qui englobe des individus, des groupes et des institutions qui entretiennent des liens plus ou moins directs, symétriques, positifs et forts ${ }^{17}$. La pluralité des formes de regroupement, des lieux d'activité et des moyens utilisés pour atteindre un objectif commun très général mais très rassembleur est une loi du genre. Dans une telle organisation multiformelle, l'institution n'est ni un lieu privilégié en soi ni le principal objectif à atteindre. Il n'y a pas, pour le réseau, d'équivalent d'un processus d'institutionnalisation. Ce processus se vit à l'échelle de groupes, même si, dans l'ensemble, le réseau recherche une légitimité sociale idéale. On l'a déjà dit, le réseau préexiste toujours, dans la mesure où il s'articule aux dimensions que les sociétés existantes se donnent. Contrairement au réseau, l'institution est une organisation plus structurée qui s'impose plutôt que se superpose à la société. Dans le réseau, les liens formels, semi-formels et informels sont tous d'égale importance, au point où si une hiérarchie de fonctions ou de pouvoir s'installe entre eux, le réseau risque de se désactiver. Le réseau n'a pas intérêt à être entièrement converti en une institution qui forcément paralyserait la partie informelle des activités du réseau, celle qui est la plus dynamique à long terme, étant donné sa grande faculté d'adaptation aux changements sociaux environnants.

Le réseau littéraire n'a pas nécessairement besoin d'une institution pour légitimer ses pratiques: le bouche à oreille plutôt que l'enseignement (transmission orale des valeurs esthétiques), le succès de lecture

16. Jacques Dubois, L'institution de la littérature, Bruxelles/Paris, Éditions Labor/Fernand Nathan, 1986 [1978], 188 p.

17. Vincent Lemieux, $\dot{A}$ quoi servent les réseaux sociaux?, op. cit. 
populaire remporté par une œuvre (légitimation populaire), l'influence de mentors intellectuels ou financiers qui ne sont pas accrédités par des institutions d'enseignement, des instances critiques ou gouvernementales (mécénat), le rassemblement désintéressé d'écrivains en herbe ou du dimanche (affinités littéraires très larges), les demandes d'aide et les échanges de compétences et d'informations sur une base amicale sont essentiellement ce qui fait la force et la faiblesse d'un réseau. Dans le réseau, contrairement à l'institution, la confiance plus que l'autorité entre les membres, entre les groupes, entre les institutions participantes et entre chacune de ces formes d'organisation sociale est une marque distinctive. Par conséquent, les luttes de pouvoir au sein d'un réseau ne se font pas à l'échelle du réseau, mais aux échelles locales, dans les différentes zones centrales ou périphériques. Aucun groupe ou aucune institution n'a d'intérêt à ce que le réseau ne serve plus ses intérêts. Étant donné que l'objectif commun est très général, par exemple, dans le cas du réseau des motards, le contrôle de la prostitution et des drogues illicites, chacune des cellules a intérêt à ce que le réseau soit fort afin qu'il conserve sa place dans toutes les sphères de la société. Les guerres de clans n'ont donc pas pour but de faire "sauter" le réseau, ou d'institutionnaliser le réseau, mais de transférer un territoire déjà existant aux membres qui font partie du réseau et qui espèrent ainsi en récolter plus d'avantages financiers ou symboliques. Des membres peuvent "disparaître ", mais rarement les zones elles-mêmes. Cette dynamique permet aux leaders des réseaux de maintenir très longtemps leur pouvoir (pensons aux parrains de la mafia italienne), contrairement aux p.d.g. et fonctionnaires de service des institutions. Ces institutions, même littéraires, respectent moins les projets individuels que les intérêts corporatifs arbitraires, c'est-à-dire imposés et régulés par les différentes lois du système judiciaire. Les réseaux préfèrent être hors-la-loi afin de ne pas perdre le contrôle de leurs activités dans et sur la société.

Voyons maintenant comment s'exercent, de manière pratique, des relations de pouvoir au sein du réseau de Casgrain. Dans le cas des regroupements semi-formels ou formels, leur leadership local est plus sujet à être remis en question que celui du chef du réseau. L'objectif commun ne fait pas l'objet de dissension, contrairement aux moyens utilisés pour l'atteindre. On y trouve des convergences ou des divergences d'opinion entre les membres lorsqu'il est question d'avoir la mainmise sur un moyen d'action littéraire incontournable, comme l'édition. Ce motif avait déjà divisé la zone centrale de Québec en 1863, entre les pro-Taché et les pro-Casgrain, autour des droits de propriété de la revue Les Soirées canadiennes, regroupement semi-formel. En 1884, un problème encore éditorial divisera la zone d'Ottawa, montrant que les luttes pour le pouvoir littéraire se passent surtout à l'intérieur de groupes. Le président T. Sterry-Hunt de la Société royale, institution littéraire logée à Ottawa, 
brisera sa promesse faite à Alfred Garneau de lui donner à corriger les épreuves des Mémoires de la Société royale du Canada, en faveur de Louis-Honoré Fréchette. Le président de la section française, Benjamin Sulte, prendra la défense de Garneau, jusqu'à démissionner de son poste; ce qui ne l'empêchera pas de demeurer la figure dominante de l'Institut canadien-français d'Ottawa. Aucun des "clans" formés par division de la zone outaouaise ne remet en question le leadership du chef du réseau, Casgrain. C'est plutôt le leadership des présidents de l'institution qui est visé. Comme en témoignent les multiples lettres alors échangées dans le réseau, l'un et l'autre clan font appel à Casgrain pour éviter l'éclatement de la zone francophone à Ottawa, qui n'aura finalement pas lieu.

\section{La dilatation spatiale et temporelle d'un réseau}

Il faut encore parler de la manière dont un réseau prend de l'expansion dans le temps et dans l'espace social. Comme on l'a déjà vu, un réseau n'a pas intérêt à trop se replier sur lui-même, surtout dans le domaine culturel. Chaque réseau a une capacité de dilatation spatiale et temporelle qui lui est propre. Néanmoins, une loi de départ semble se dégager de l'observation de réseaux de correspondance aussi différents que celui de Casgrain, d'Alfred DesRochers, de Camille Roy, de La Relève, de l'Hexagone ou des intellectuels de La Nouvelle Revue française, de la période de la République des lettres au $\mathrm{xvII}^{\mathrm{e}}$ siècle québécois ou de la Révolution tranquille ${ }^{18}$. Un peu comme une galaxie dans l'univers, un réseau n'a, de fait, ni véritable commencement ni fin. Une de ses caractéristiques, par rapport aux groupes et aux institutions qui le composent, est qu'il est en quelque sorte une "énergie renouvelable" qui peut servir à d'autres fins que les précédentes, mais qui sert toujours coûte que coûte. En ce sens, il y a des réseaux potentiels, virtuels ou des zones au sein d'un réseau qui n'existent pas autrement tant et aussi longtemps qu'elles ne sont pas activées. Dans le domaine littéraire, les réseaux intertextuels en sont probablement l'exemple le plus frappant: des cuvres littéraires qui existent déjà peuvent n'être "activées" qu'un siècle plus tard, récupérées pour la création de nouvelles ceuvres. Vincent Lemieux définit ainsi le réseau potentiel: "réseau fait de tous les participants et de leurs connexions, susceptibles d'entrer en action dans un contexte donné ${ }^{19}$ ". Ce qui vaut à l'échelle d'une oeuvre vaut aussi à l'échelle d'un réseau littéraire en partie ou en totalité.

18. Pierre Rajotte (dir.), Lientx et réseanx de sociabilité littêraire au Québec, Québec, Éditions Nota bene, 2001, 335 p., comprenant, entre autres, les travaux de Richard Giguère sur DesRochers et de Nancy Houle sur La Relève; Benoît Melançon (dir.), Penser par lettre, Montréal, Fides, 1998, 375 p., collectif dans lequel on trouve sur les réseaux des articles de Michel Lacroix, Martine Fisher, Michel Biron, Jane Everett, Pierre Popovic, Micheline Cambron, Bernard Andrès et Benoît Melançon.

19. Vincent Lemieux, $\dot{A}$ quoi servent les réseau x sociaux? op. cit., p. 8 
Ainsi, Casgrain n'a pas créé un réseau mais l'a activé. Des connexions existent déjà entre des écrivains ou des milieux littéraires au moment où Casgrain entreprend de créer un programme et des activités pour les activer au maximum. Elles sont plus ou moins faibles et fiables. Depuis la Conquête, les écrivains n'ont pas encore appris à travailler ensemble, tels les F.-X. Garneau, É. Parent, P.-J.-O. Chauveau et O. Crémazie. Le dynamisme de Casgrain lui vaut d'être appelé par les membres du réseau, "Cher ami", "Cher Monsieur L'Abbé", "Très cher ami ", "Cher Maître" ou "Père de la littérature nationale ${ }^{20}$ ", au fur et à mesure que le réseau se dilate et que la grande influence de Casgrain se confirme. Les écrivains qui se réunissent pour fonder et collaborer au Foyer canadien (18631866) se connaissent déjà tous, mais se donnent pour la première fois, en dehors des engagements politiques plus ou moins anonymes, l'occasion de faire cuvre commune: "Nous avons le plaisir d'annoncer au public que le Foyer canadien comptera parmi ses patrons et collaborateurs les littérateurs dont suivent les noms, au nombre desquels se trouvent plusieurs de nos écrivains canadiens les plus distingués: Messieurs Étienne Parent, F.-X. Garneau, Ph. Aubert de Gaspé, Patrice Lacombe, F.-M. Derome, les abbés C. Trudelle, C. Laverdière, C. Legaré, Messieurs J.-M. LeMoine, C. Laberge, Félix-G. Marchand, A.-A. Boucher, Alfred Gar-neau, J. Auger, Ernest Gagnon, E.-L. de Bellefeuille, Henri-T. Taschereau, L.-H. Fréchette et L.-P. LeMay". Et cette liste est signée par: "L'abbé J.-B.-A. Ferland, Président, Archevêché; L.-J.-C. Fiset, Rue Petit Richmond; A. Gérin-Lajoie, Assemblée législative; L'abbé H.-R. Casgrain, Presbytère; F.-A.-H. La Rue, Secrétaire, Rue Saint-François ${ }^{21}$ ". Les noms sont spontanément suivis du lieu où l'on peut facilement rencontrer les responsables si l'on désire contribuer à la cause qu'ils défendent. Sur la page-titre de la livraison, on note aussi cette autre adresse utile: "Québec, Bureaux du "Foyer Canadien", Coin des rues Sainte-Anne et des Jardins". Le réseau potentiel s'active, ouvre ses portes.

Dans ses Souvenances canadiennes, Casgrain explique très bien dans quel contexte furent activées les relations déjà existantes entre ces écrivains: "Les esprits observateurs auraient pu dès lors le ressentir", dit-il. Nous citons ce passage inédit un peu long auquel on se référera aussi plus loin:

La ville de Québec, siège du gouvernement, était en même temps le centre intellectuel du pays. Chaque jour je pouvais être en relation avec des hommes tels que nos historiens Garneau et Ferland, le savant et modeste abbé Laverdière de l'Université Laval, Octave Crémazie, Étienne Parent, Chauveau, de Gaspé, Gérin-Lajoie, J. C. Taché, le Dr LaRue, A. E. Aubry, professeur de

20. Manon Brunet, "Henri-Raymond Casgrain et la paternité d'une littérature nationale", Voix et images, $n^{\prime \prime} 65$, hiver 1997, p. 205-224. Ces appellations reviennent souvent dans la correspondance.

21. "Prospectus", Le Foyer canadien, vol. I, 1863, p. 8-9. Les mots sont en italique dans le texte original. 
droit romain et rédacteur du Courrier du Canada, sans parler de bien d'autres qu'il serait trop long de nommer. C'était, on le voit, la fleur de la société canadienne-française au point de vue intellectuel. Rarement avait-elle été aussi intéressante à voir de près, parce qu'on y sentait flotter un souffle d'inspiration d'où allaient surgir toute une génération de littérateurs nouveaux et des œuvres d'une réelle originalité. Les esprits observateurs auraient pu dès lors le pressentir. [...]

La génération de 1860 , toute frémissante de la récente victoire lobtention du gouvernement responsable grâce à la lutte menée par Sir Louis LaFontaine], aspirait à de nouveaux triomphes. Les hommes de lettres rêvaient la création d'une littérature nationale. Ils avaient à cour de donner un démenti à l'assertion de lord Durham qui avait soutenu dans son fameux rapport que les Canadiens-Français n'étaient pas véritablement un peuple, parce qu'ils n'avaient pas de littérature. Dans les cercles littéraires de Québec, c'était la question qui revenait le plus souvent et qui passionnait les esprits. Il n'y avait pas de réunions concertées. On se rencontrait par hasard un peu partout, par petits groupes de trois ou quatre, quelquefois plus, tantôt à la bibliothèque du Parlement, autour du bureau de Gérin-Lajoie, alors bibliothécaire, tantôt à la rédaction du Courrier du Canada, rue Buade, tantôt à la librairie d'Octave Crémazie, rue de la Fabrique, voire même chez moi au presbytère de NotreDame. Les plus assidus à ces réunions, ou du moins ceux qui se rencontraient le plus souvent, soit dans un lieu soit dans un autre, étaient Octave Crémazie, Chauveau, Taché, Gérin-Lajoie, LaRue ${ }^{22}$.

L'aspect informel de ces premières rencontres, qui conduiront plus tard, avec presque les mêmes protagonistes, à la création de la Société royale du Canada, est à remarquer. Les échanges formels et informels sont des particularités des réseaux, lesquels refusent de se laisser enfermer dans une seule organisation, institution. Ferland, Crémazie et Casgrain seront les observateurs qui auront eu le meilleur pressentiment sur le degré de "potentialité" que recélaient ces connexions fortuites. Après l'exil de Crémazie en 1862, la mort en 1865 de J.-B.-A. Ferland, son "Mentor ${ }^{23}$ " et celle de l'historien national F.-X. Garneau au début de 1866, Casgrain prendra la relève et réactivera à sa manière le réseau qui risque par ces pertes intellectuelles importantes de retomber au point mort: il publie dans Le Foyer canadien, en 1866, la première version de son programme littéraire: "Le mouvement littéraire en Canada". Nous verrons plus loin les conséquences très importantes qui découlent de l'intervention de Casgrain dans le renouvellement du programme littéraire.

22. Musée de la civilisation de Québec (MCQ), Fonds Casgrain, Henri-Raymond Casgrain, Souvenances canadiennes, tome III, O445, p. 32 et 43-44.

23. L'abbé Jean-Baptiste-Antoine Ferland fut, avec Crémazie et l'abbé Pierre Bouchy, l'un des principaux mentors de Casgrain: "Parmi ces conseillers, il en est un à qui jai voué une reconnaissance éternelle, c'est l'abbé Ferland, le savant annaliste, l'aimable causeur, qui rappelait Fénelon par la douceur du caractère, l'élévation d'esprit et le fluide sympathique qui s'échappait de sa personne et de ses discours. $[\ldots]$ nous pouvions chaque jour nous voisiner. Sans avoir l'air d'y toucher, sans même y songer probablement, il s'était fait mon Mentor ", ibid., p. 40. 
La dilatation du réseau de Casgrain dépend en grande partie des activités que le chef mène pour réaliser ses propres oeuvres, surtout dans le domaine historique. Sans modèle et sans stimulation constante, le réseau s'affaiblirait. Le sous-titre du Foyer canadien n'était-il pas: "Recueil littéraire et bistoriquen? Poète et critique, l'abbé est avant tout un historien. Chaque occasion de rencontrer des archivistes, des littérateurs, des familles, des éditeurs, des libraires ou des académiciens en est une qui sert à la fois son ouvre, au sens restreint, et son CEuvre, au sens large, d'animateur du réseau littéraire québécois. Afin de faire (de) la littérature nationale, l'historien cherche donc de nouveaux matériaux historiques pour lui, pour Marmette, pour Parkman, pour Wrong, enfin pour tous ceux et celles qui répondront aux exigences de réalisation du projet de littérature nationale. Nous ne détaillerons pas ici ces exigences, car elles sont connues ${ }^{24}$. Sa recherche de matériaux et de "talents" ne connaît pas de bornes: Casgrain y consacre tout son temps, tout son argent, toute son énergie intellectuelle et agrandit son territoire de recherche au cours de ses rencontres, de ses voyages, de sa correspondance. L'amplification de la correspondance, reçue et envoyée au fil des années, rend bien compte de ce phénomène de dilatation du réseau.

\section{Répartition de la correspondance de Henri-Raymond Casgrain, 1851-1904, selon les activités du réseau}

\section{Période 1 : 1851-1886 (35 ans - 1600 lettres - $32 \%$ du corpus)}

Du début de la vocation ecclésiastique et littéraire de Casgrain jusqu'à la fin de l'Affaire Taché vs Casgrain (Rome).

Ce sont des années d'implantation d'une infrastructure utile à la production, à la diffusion et à la légitimation de la littérature nationale au Québec et à l'étranger. Cette infrastructure se développe au même rythme que le réseau. La zone centrale s'affiche, s'organise et développe une connexité forte. Les membres de cette zone centrale du réseau sont concentrés à Québec et à Ottawa et, comme ils se voient régulièrement pour créer et faire fonctionner l'infrastructure, ils ont moins affaire à s'écrire. D'où le peu de lettres pour une période aussi longue et active. Les zones périphériques américaine et française servent de relais.

Période 2: 1887-1895 (8 ans - 1800 lettres - $36 \%$ du corpus)

De l'année où Casgrain obtient le titre de Professeur titulaire à l'Université Laval jusqu'à la fin de ses travaux pour la Collection des manuscrits du Maréchal de Lévis (12 vol.).

Ce sont les années de voyages répétés et de consécration (Casgrain obtient un prix de l'Académie française en 1888). En l'espace de seulement 8 ans, les

24. Maurice Lemire, La littérature québécoise en projet an milieu du $19^{\prime \prime}$ siècle, Montréal, Fides, 1993, 276 p.; Réjean Beaudoin, Naissance d'une littérature: essai sur le messianisme et les débuts de la littérature canadienne-française, 1850-1900, Montréal, Boréal, 1989, 209 p.; Maurice Lemire, Denis Saint-Jacques (dir.), La vie littéraire au Québec, tome in: Un peuple sans littérature ni bistoire, 1840-1869, Québec, Les Presses de l'Université Laval, 1996, 671 p.; id., tome Iv: Je me souviens, 1870-1894, Québec, Les Presses de l'Université Laval, 1999, 669 p. 
échanges d'informations, de livres et d'iclées pullulent au sein du réseau qui s'étend géographiquement et intellectuellement tous azimuts, d'où le grand nombre de lettres conservées pour une si courte période. La zone acadienne est activée et la zone française s'étend considérablement.

Période 3 : 1896-1904 (8 ans - 1600 lettres - $32 \%$ du corpus)

De la publication de la dernière édition des Futures complètes de Casgrain jusqu'à sà mort.

Dilatation maximale du réseau tel qu'activé par Casgrain et contraction progressive. Avec la mort de Parkman (1893) et celle de Rameau de Saint-Père (1899), le réseau perd graduellement ses relais avec les zones américaine et française. Cependant, une ouverture se fait du côté canadien-anglais avec la venue du jeune historien George $M$. Wrong de l'Université de Toronto. Aveugle, Casgrain ne voyage presque plus. La correspondance assure presque à elle seule le maintien du réseau à distance. L'heure est au bilan, aux sonvenances et à la réévaluation des travaux du réseau québécois par des critiques étrangers, tel Charles ab der Halden, ou locaux qui prendront de plus en plus de place au sein des institutions d'enseignement, tel Camille Roy, et de nouveaux écrivains, comme ceux de l'École littéraire de Montréal qui défendent un nouveau programme littéraire.

\section{La dilatation idéologique d'un réseau}

On a vu, dans la citation ci-avant de Casgrain, que le contexte idéologique d'après 1840 avait amené les intellectuels à s'activer autour d'un projet commun: "Les hommes de lettres rêvaient la création d'une littérature nationale. Ils avaient à cou de donner un démenti à l'assertion de lord Durham qui avait soutenu dans son fameux rapport que les Canadiens-Français n'étaient pas véritablement un peuple, parce qu'ils n'avaient pas de littérature. " Or, il ne suffit pas pour le fonctionnement à long terme d'un réseau culturel que les membres partagent un objectif précis, si louable et justifié soit-il par la trame de la conjoncture sociale. La tolérance du réseau de Casgrain vis-à-vis cles différences idéologiques peut donc paraître surprenante, mais elle est inévitable afin d'assurer à la fois la cohésion et l'ouverture de son réseau, en d'autres mots, sa survie. Surtout, cette tolérance est la meilleure garantie que l'objectif sera atteint un jour ou l'autre, et maintenu, et ultérieurement inscrit dans la mémoire collective. Dans les réseaux culturels, les débats et les divergences d'opinion assurent une pérennité au projet, même si le réseau est sujet à renouveler sa configuration avec le temps. Effectivement, le projet de littérature nationale au Québec traversera bien des générations littéraires jusqu'à la nôtre, et l'histoire littéraire est encore curieuse de savoir comment les choses ont vraiment commencé.

Cette dilatation idéologique explique l'exceptionnelle longue durée et la réelle influence de l'homme et de son réseau. Casgrain n'ignore pas ces différences, il les critique parfois sévèrement (comme dans le cas des œuvres de Parkman), mais il les encourage et les respecte comme pas un (Casgrain voudra que l'Université Laval décerne à Parkman un doctorat 
bonoris causa). Car il lui importe plus qu'on fasse l'histoire du Canada français, d'une manière ou d'une autre, que la façon de la faire. Ainsi, Casgrain admet dans son réseau, contrairement à ce qui se passe dans le réseau ultramontain des Laflèche, Taché, Tardivel et Routhier, des écrivains de tous statuts ou allégeances: qu'on soit laiic ou religieux, femme (Laure Conan) ou féministe (Clarisse Coignet), à peine lettré (Arthémise Tapin) ou érudit, protestant (Parkman) ou catholique, anglophone (G. M. Wrong) ou francophone (Rameau de Saint-Père), libéral (L. Fréchette) ou conservateur (J.-C. Taché), romancier (J. Marmette) ou poète (A. Garneau). La libéralité de Casgrain est très évidente. De plus, la correspondance de Casgrain nous montre très clairement que son réseau est surtout d'allégeance libérale. Chez Casgrain, son statut d'abbé nous a fait trop souvent confondre l'habit et le moine. La première version de son programme littéraire que l'on cite toujours, sans le contexte évoqué tantôt, n'y est pas pour rien non plus. Il faut lire aussi bien d'autres textes de Casgrain, comme "Notre passé littéraire et nos deux historiens", prononcé à la séance inaugurale de la Société royale du Canada en $1882^{25}$, pour comprendre comment évolue sa pensée intellectuelle, et surtout lire sa correspondance, qui montre l'influence que le réseau libéral, tant clérical et laïc, a sur son chef. Voici le bilan que Casgrain dresse de l'influence de son réseau sur lui :

Les recherches historiques que j'avais commencées à Paris et que je repris à mon arrivée me mirent bientôt en rapport avec divers hommes de science, des archivistes, des antiquaires, des archéologues, toute une société d'érudits, la plus attachante que puisse rêver un homme d'étude [...]. La fréquentation cle ces hommes d'élite m'a initié mieux qu'aucune lecture au mouvement intellectuel européen. Elle m'a ouvert des horizons inconnus et procuré les jouissances les plus intenses et les plus durables que j'ai rapportées de mes voyages ${ }^{26}$.

De grandes figures libérales s'affichent dans le réseau de Casgrain: que ce soit $\mathrm{M}^{\mathrm{gr}}$ Elzéar-Alexandre Taschereau que les ultramontains taxeront de franc-maçon alors que Casgrain sera le principal instigateur de son cardinalat à Rome en 1886, que ce soit Wilfrid Laurier, Honoré Mercier ou Félix-Gabriel Marchand qui cautionneront généreusement sous leur gouvernement libéral, toutes les initiatives prises par Casgrain au nom de la littérature nationale, autant sinon plus que que sous le conservateur Gédéon Ouimet. L'abbé n'est pas un Buies ni un Dessaulles, mais il est à coup sûr aussi libéral qu'Étienne Parent et que F.-X. Garneau l'étaient. $\mathrm{M}^{\mathrm{gr}}$ Bourget l'a à l'œeil: lors de la sortie en livre de Vies des

25. Henri-Raymond Casgrain, "Notre passé littéraire et nos deux historiens", L'Opinion publique, vol. XIII, $\mathrm{n}^{\circ} 23,8$ juin 1882, p. 268. Conférence lue devant la Société royale du Canada le 26 mai 1882.

26. MCQ, Fonds Casgrain, Henri-Raymond Casgrain, Souvenances canadiennes, tome II, O445, p. 113. 
saints, il reproche vertement à Casgrain d'y avoir publié des chants mis à l'Index $^{27}$. $\overline{\mathrm{A}}$ l'instar de Pierre Bouchy ${ }^{28}$, son mentor romantique depuis l'époque de ses études au Collège de Sainte-Anne-de-la-Pocatière, Casgrain préfère Montalembert à Veuillot, deux intellectuels qu'il a rencontrés en 1858 et 1867: "Le contraste ne pouvait être plus prononcé [...] Montalembert représentait la société moderne avec son amour de la liberté, du progrès, du parlementarisme, en un mot, c'était le chef libéral; tandis que Veuillot était l'homme de l'âge gothique, partisan des traditions démodées, du droit divin, de l'absolutisme ${ }^{29}$." Une lettre de la féministe Clarisse Coignet résume à elle seule comment le "socialiste "Casgrain était perçu auprès des membres les plus libéraux de son réseau à la fin du siècle:

Puisque vous êtes socialiste [...], je me permets de vous envoyer la biographie d'un de mes cousins et amis [...]. Si vous êtes libre, mercredi prochain, à 7 heures $1 / 4$, combien je vous saurais gré de venir partager un dîner très modeste, avec trois ou quatre amis, pas davantage. Je suis sûre que vous les intéresserez beaucoup et que vous les étonnerez un peu, votre façon de comprendre le catholicisme étant tellement plus large que celle de la plupart de nos prêtres et laïques français. Et puis, vous nous faites tant de bien en nous montrant dans le Canada une nouvelle France.

Mille aimables souvenirs.

\section{Coignet}

P.-S. Le dîner sera tout à fait sans cérémonie, sans habit ${ }^{30}$.

Ainsi, le réseau prend de l'expansion au fur et à mesure que les connexions entre les membres du réseau s'activent, s'intensifient, se renforcent et se multiplient grâce au dynamisme de Casgrain. Certaines connexions se désactivent, comme après l'épisode du conflit entre Joseph-Charles Taché et Casgrain durant la première année de direction des Soirées canadiennes en 1861, conflit qui donnera lieu d'ailleurs à la création d'une revue littéraire parallèle, Le Foyer canadien, qui remportera un succès foudroyant dès 1863 avec ses 2000 abonnés. Taché ne pardonnera jamais à Casgrain d'être devenu le Mentor du réseau québécois et jusqu'en 1886, année où il perd son procès à Rome contre Casgrain pour

27. Voir les lettres envoyées à Casgrain par Ignace Bourget et son Secrétaire Joseph-Octave Paré en 1865, 1866 et 1870, MCQ, Fonds Casgrain, Lettres diverses, tome II, O449, n 16, 33, 44; tome III, O450, $\mathrm{n}^{\circ} 71$; tome $\mathrm{v}, \mathrm{O} 451, \mathrm{n}^{\circ} 22,26,27,57$. La beauté de la vie des saints " parut d'abord anonymement dans Le Courrier du Canada, en mars-avril 1865 , ensuite, en livre sous le titre de Vies des saints pour tous les jours de l'année, recueillies des meilleurs auteurs, Ottawa, George-É. Desbarats, $1868,750 \mathrm{p}$.

28. Lettre de Charles de Montalembert à Pierre Bouchy, 19 mars 1858, dont l'original est perdu, mais citée dans MCQ, Fonds Casgrain, Henri-Raymond Casgrain, Souvenances canadiennes, tome II, O444, p. 146-147.

29. MCQ, Fonds Casgrain, Henri-Raymond Casgrain, Sotwenances canadiennes, tome II, O444, p. 151.

30. MCQ, Fonds Casgrain, Lettres diverses, lettre cle Clarisse Coignet à Henri-Raymond Casgrain, 12 [décembre] 1897, tome xxl, O468, n 67. 
une question de droits d'auteur qu'il est le seul à revendiquer, les liens avec Taché seront extrêmement ténus, négatifs dirait $\mathrm{V}$. Lemieux, de même que tous les liens indirects entre des tiers appartenant soit au réseau de Casgrain, soit à celui de Taché. Ainsi, en 1872, J. Marmette et Casgrain silhouetteront Taché ${ }^{31}$, mais la réplique viendra d'un membre du réseau ultramontain de Taché, A.-B. Routhier, qui signera en 1873, sous le pseudonyme de Jean Piquefort, un portrait peu flatteur de Casgrain ${ }^{32}$ en se moquant de son titre de "père de la littérature nationale".

\section{Voir le littéraire}

Il faut conclure sur une difficulté méthodologique de taille dans l'analyse des pratiques des réseaux : comment discerner les échanges littéraires parmi tous les autres? Plusieurs formes d'échanges sont observables à travers la correspondance. Que les regroupements soient informels, semiformels ou formels, les échanges littéraires existent pour reconnaître des liens d'appartenance au réseau, pour faire circuler de l'information, pour apporter de l'aide, pour mobiliser du capital social, pour relier les agents littéraires entre eux et pour contrôler les politiques littéraires, les six raisons d'existence d'un réseau selon Vincent Lemieux ${ }^{33}$. La nature de ces échanges est très variée, mais ils sont reconnaissables parmi tous les autres de par leur finalité littéraire. La question de la finalité littéraire d'une activité est cruciale pour le repérage de ce qui est littéraire dans le réseau. Sans ce concept d'analyse, le chercheur s'expose à être incapable de distinguer l'essentiel de l'accessoire, à perdre de vue une problématique de recherche littéraire et à de graves anachronismes.

Écrire, publier ou lire un livre n'est pas en soi une activité littéraire. Tout individu dans une société peut écrire, publier ou lire un livre pour autant qu'il soit alphabétisé. Rien n'empêche de faire la lecture d'un livre pour faire de la propagande religieuse, d'en écrire pour compiler des recettes ou d'en acheter pour décorer les rayons d'une bibliothèque. C'est ce pourquoi cet individu s'adonne à une activité qui définit le sens de son activité et non comment il s'active. Le moyen utilisé pour faire de la littérature peut être classique ou inusité, mais l'objectif reste le même. Par exemple, on sait qu'une activité en apparence aussi peu littéraire que de

31. Placide Lépine (pseud. de Joseph Marmette et de Henri-Raymond Casgrain), "Silhouettes litteraires: Joseph-Charles Taché "L'Opinion publique, vol. III, $\mathrm{n}^{\circ} 7$, 15 février 1872, p. 74 . Taché est le premier de la série. Sur toute cette affaire, voir Manon Brunet, "Silhouettes", "portraits" et "profils": la critique biographique de "nos hommes de lettres" au $19^{\mathrm{e}}$ siècle", Annette Hayward et Agnès Whitfield (dir.), Critique et littérature québécoise, Montréal, Triptyque, 1992, p. 97-107.

32. Jean Piquefort (pseud. de Adolphe-Basile Routhier), "Portraits et pastels littéraires: L'abbé Casgrain", Le Courrier du Canada, vol. VI, n' 140,10 janvier $1873 ; \mathrm{n}^{\circ} 141$, 13 janvier; $n^{\circ} 142,15$ janvier; $n^{\prime 3} 143,17$ janvier.

33. V. Lemieux, À quoi servent les réseaux sociaux?, op. cit. 
voyager peut avoir comme finalité de produire du littéraire. Des activités identiques, qui seront considérées comme littéraires dans un réseau littéraire, peuvent très bien ne devenir que des moyens parmi d'autres de produire du politique ou du religieux. De plus, nous n'avons pas à attendre que des pratiques soient vraiment littéraires selon nos critères actuels, ou autonomisées pour voir du littéraire. La littérature se fait ni plus ni moins qu'en fonction des critères formels et idéologiques promus par le réseau.

Revenons au voyage. Les exemples sont nombreux dans la littérature de l'usage du voyage comme moyen de produire du littéraire. Dans la littérature québécoise, à commencer par François-Xavier Garneau qui en a tiré un récit de voyage en France et en Angleterre; Casgrain, lui, trouve dans le voyage un moyen privilégié de renforcer son réseau littéraire; les écrivains du Nigog s'inspireront abondamment de leurs voyages européens pour écrire leurs cuvres qualifiées d'"exotiques", tout comme Alain Grandbois et nos auteurs actuels se servent du voyage comme d'un moyen de promotion littéraire à l'étranger, dans les salons du livre. Cette énumération est presque banale. Or, d'en tenir compte dans une analyse approfondie des pratiques littéraires, l'est beaucoup moins. Toutes ces activités sont littéraires à commencer par le voyage lui-même. Le littéraire est plus que la littérature, tout comme le politique est irréductible à la politique. La littérature n'est qu'une manifestation, qu'un résultat, parmi bien d'autres aussi importants, de l'activité littéraire. Là, comme ailleurs, la fin ne peut être confondue avec le moyen. Par conséquent, les lettres que nous avons retenues pour l'édition de la correspondance casgrainienne ne sont pas nécessairement des œuvres littéraires en elles-mêmes ni ne parlent uniquement des ouvres littéraires au sens restreint. Néanmoins, ce sont des écrits qui attestent hors de tout doute des activités de tout genre qui sont mises à contribution dans le réseau pour soit produire, soit diffuser, soit encore légitimer du littéraire (i.e. des ouvres littéraires au sens large), selon l'borizon d'attente fixé par le réseau. Malgré cette précaution méthodologique de base, qui est fort utile pour distinguer l'essentiel de l'accessoire, nous n'avons pas toujours respecté cette règle dans le cas de correspondances aux finalités religieuse, politique, familiale ou amicale, ou presque amoureuse, car nous avons considéré que certaines lettres éclairaient grandement des pans biographiques inconnus de Casgrain. Mais, chaque fois, nous l'avons fait en connaissance de cause.

Finalement, le réseau littéraire, à l'instar de tout réseau culturel, consacre l'essentiel de ses activités à se trouver une légitimité, aujourd'hui comme hier. Toutes les sociétés accordent une faible légitimité sociale et

34. Pierre Bourdieu, avec Lö̈ J. D. Wacquant, Réponses; pour une anthropologie réflexive, Paris, Seuil, 1992, p. 142-143.

35. Ibid. Souligné dans le texte original. 
économique aux réseaux culturels par rapport aux réseaux de parenté, aux réseaux politiques ou aux réseaux économiques. Autrement dit, les réseaux légitimés exercent sur le réseau culturel une réelle "violence symbolique ". J'emprunte ce terme à Bourdieu, qui, lui, l'utilisait dans son analyse de la domination en général, pour désigner "le fait d'accepter cet ensemble de présupposés fondamentaux, pré-réflexifs, que les agents sociaux engagent par le simple fait de prendre le monde comme allant de soi ${ }^{34}$. Il en concluait que "De toutes les formes de "persuasion clandestine", la plus implacable est celle qui est exercée tout simplement par l'ordre des choses ${ }^{35}$. Dans l'ordre des choses, la culture est toujours un "luxe" placé au-dessus des besoins élémentaires sociaux, selon la fameuse pyramide de Maslow. Même si la configuration des réseaux culturels peut être semblable en plusieurs points à celle des autres réseaux, le fait que dans un réseau culturel les relations tissées aient comme objectif principal et permanent la recherche de la légitimation auprès des autres réseaux, il en résulte un réseau aux contours plus que fragiles, très flous et à l'évolution difficilement prévisible. Malgré les apparences, cela vaut pour tout réseau culturel, même ceux qui défendent l'art pour l'art, même pour l'institution littéraire qui n'est, ne l'oublions pas, qu'un lieu parmi tous les autres au sein d'un réseau littéraire à configurations multiples, qu'il soit plus ou moins bien constitué et légitimé. Vu d'une manière plus positive, on dirait que le dynamisme du réseau littéraire ne dépend ni plus ni moins que de ces contraintes propres à son genre. S'impose alors une méthodologie d'analyse qui permet l'observation à la fois de ce dynamisme et de ces contraintes. 